\title{
Bloqueo anestésico de los nervios intercostales T6-T11 en un canino sometido a escisión quirúrgica de masa tumoral ubicada en pared abdominal cráneo-ventral. Reporte de caso
}

\author{
Diego Alejandro Ospina-Argüelles $\mathbb{D}^{1}$, Edwin Fernando Buriticá-Gaviria ${ }^{\mathbb{D}}{ }^{1}$, Diego \\ Fernando Echeverry-Bonilla (id ${ }^{1}$.
}
1 Grupo de Investigación en Medicina y Cirugía de Pequeños Animales, Facultad de Medicina Veterinaria y Zootecnia, Universidad del Tolima, Ibagué, Colombia.

\section{daospina@ut.edu.co}

\author{
Recibido 24 de Septiembre de 2016 y Aprobado 23 de Noviembre de 2016 y \\ actualizado 22 de Junio
}

DOI: 10.17151/vetzo.2017.11.1.7

RESUMEN: Se expone el manejo anestésico realizado a un canino hembra de raza Golden Retriever de 8 años de edad sometida a escisión quirúrgica de un tumor maligno localizado en la pared del abdomen cráneo-ventral; para lo cual se efectuó el bloqueo anestésico bilateral de los nervios intercostales T6-T11 empleando marcas anatómicas de superficie como técnica de neurolocalización, y usando bupivacaína $(0,5 \%)$ como anestésico local. Lo anterior como parte de un protocolo de anestesia multimodal que también incluyó acepromacina, meloxicam, tramadol, propofol e isoflurano en $\mathrm{O} 2$ al $100 \%$. Los valores de presión arterial media y frecuencia cardiaca fueron evaluados intraoperatoriamente; el dolor postoperatorio fue evaluado aplicando para ello la escala compuesta del dolor de Glasgow. Las variables cardiorrespiratorias intraoperatorias evaluadas se mantuvieron estables durante todo el procedimiento quirúrgico. La valoración postoperatoria del dolor evidenció una adecuada cobertura analgésica. El bloqueo intercostal empleado en este paciente como parte de un protocolo de anestesia multimodal permitió minimizar el consumo de isoflurano, y evitó el empleo de analgesia de rescate en el periodo intraoperatorio, así como la necesidad de administrar anestesia suplementaria en el postoperatorio evaluado. El bloqueo de los nervios intercostales T6-T11 por medio de la técnica de marcas anatómicas de superficie, sumado al protocolo multimodal empleado, permitió obtener una cobertura analgésica eficaz, económica y segura en el paciente evaluado. Esta técnica podría ser considerada como una alternativa frente al bloqueo epidural tradicionalmente realizado en procedimientos quirúrgicos que involucran la pared abdominal.

Palabras clave: analgesia, bloqueo nervioso, epidural, perros

\section{Anesthetic block of the T6-T11 intercostal nerves in a canine underwent a surgical excision of a tumor mass located in the cranio-ventral abdominal wall}

\footnotetext{
ABSTRACT: The anesthetic management of an 8-year-old Golden Retriever female canine submitted to surgical excision of a malignant tumor located in the wall of the cranio-ventral abdomen was exposed; for which the T6-T11 bilateral intercostal nerves anesthetic block was performed using anatomical surface marks as
} 
neurolocalization technique, and using bupivacaine $(0.5 \%)$ as local anesthetic. This as part of a multimodal anesthetic protocol that also included acepromazine, meloxicam, tramadol, propofol and isoflurane in $100 \% \mathrm{O} 2$. The values of arterial pressure and heart rate were evaluated intraoperatively; postoperative pain was evaluated applying the composite Glasgow pain scale. Intraoperative evaluated cardiorespiratory variables remained stable throughout the surgical procedure. Postoperative pain assessment showed adequate analgesic coverage. The intercostal block used in this patient as part of a multimodal anesthesia protocol allowed to minimize the consumption of isoflurane, and avoided the use of rescue analgesia in the intraoperative period, and the need to administer supplemental anesthesia in the postoperative period. The blockade of the intercostal nerves T6-T11 by means of the surface anatomical marks technique, added to the multimodal protocol used, allowed to obtain effective analgesic coverage, economical and safe in the evaluated patient. This technique could be considered as an alternative to the epidural block traditionally performed in surgical procedures involving the abdominal wall.

Key words: analgesia, dogs, epidural, nerve block

\section{Introducción}

Se ha establecido que la cirugía abdominal provoca una considerable estimulación nociceptiva (Hellyer et al., 2007), donde el dolor referido por pacientes humanos sometidos a este tipo de cirugía se origina principalmente a nivel de la incisión de la pared abdominal (Melzack \& Wall, 1999). La magnitud del dolor experimentado depende en parte de la agresividad de la intervención quirúrgica realizada (Fox, 2014), siendo en este sentido la cirugía oncológica particularmente agresiva.

Recientemente se ha propuesto el bloqueo anestésico del plano abdominal transverso (TAP block) como una alternativa eficaz para proveer analgesia durante la realización de cirugías que comprometan la pared abdominal (Schroeder et al., 2011; Bruggink et al., 2012). Sin embargo, el TAP block no permitiría obtener una cobertura analgésica suficiente para la realización de procedimientos quirúrgicos efectuados en el aspecto más craneal de la pared abdominal (Schroeder et al., 2011).

Se sugiere que el bloqueo de los nervios intercostales (BNI) caudales puede complementar la cobertura analgésica obtenida a partir de la realización del TAP block, o servir como única técnica anestésica loco-regional para procedimientos quirúrgicos realizados en el aspecto más craneal de la pared abdominal en humanos o animales (Finnerty et al., 2010; Portela et al., 2014; Echeverry et al., 2015). El efecto analgésico de este bloqueo ha sido demostrado en perros bajo condiciones clínicas y experimentales (Trębacz et al., 2011; Echeverry et al., 2015).

El objetivo de este reporte de caso es describir el efecto analgésico perioperatorio obtenido a partir de la realización del bloqueo bilateral de los nervios intercostales T6- 
T11 en un perro sometido a escisión quirúrgica de una masa tumoral localizada en la pared cráneo-ventral del abdomen.

\section{Evaluación del paciente}

\section{Anamnesis}

Fue presentada al Servicio de Cirugía de la Clínica de Pequeños Animales de la Universidad del Tolima una hembra canina de raza Golden Retriever de 8 años de edad, $33,7 \mathrm{~kg}$ y una condición corporal 4/5, por presentar una masa de 8 x $5 \mathrm{~cm}$ localizada en el abdomen cráneo-ventral, con un curso de aparición de 30 días (Figura 1).

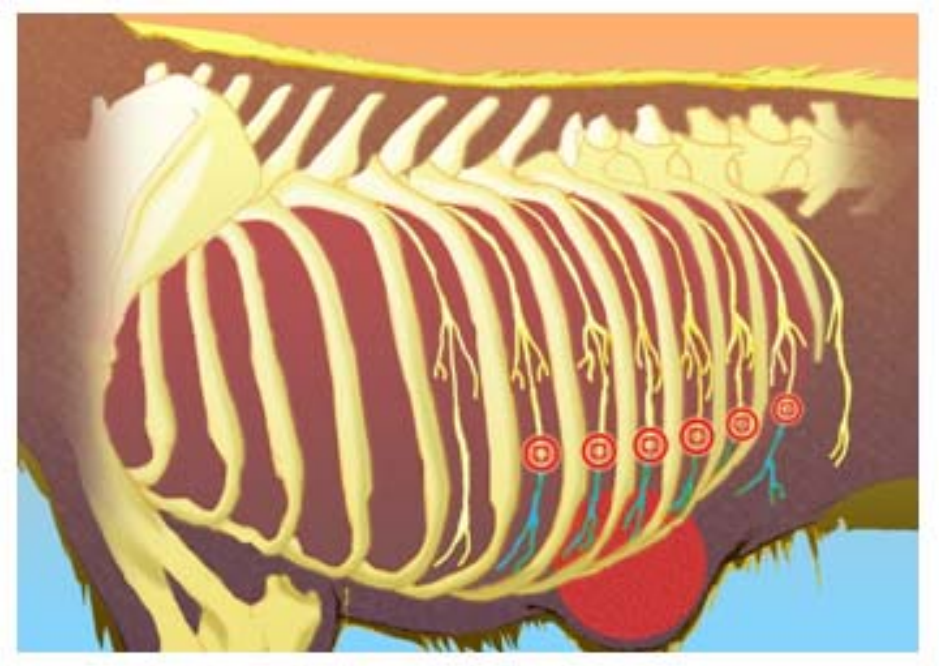

Figura 1. Diagrama que ilustra la localización de la masa tumoral y los sitios de bloqueo de los nervios intercostales T6-T11.

\section{Plan diagnóstico y terapéutico}

Se realizó un hemograma de segunda generación, examen general de orina y bioquímica sanguínea básica con resultados dentro de los rangos normales para los analitos evaluados. Posteriormente se efectuó una biopsia con aguja fina que reveló la presencia de proliferación de fibroblastos bien diferenciados indicando una fibroplasia reactiva, proceso compatible con un fibrosarcoma bien diferenciado. Este resultado sugirió la posible malignidad de la masa, por lo que se procedió a realizar una escisión quirúrgica de la misma por medio de una resección tisular amplia. Dicho procedimiento quirúrgico incluyó un margen de cinco centímetros a cada extremo de la masa y un $\mathrm{cm}$ de profundidad, con resección de la porción del músculo recto abdominal inmediatamente inferior al tejido a escindir de cada hemiabdomen. De igual manera, la glándula mamaria torácica craneal izquierda fue resecada acorde a los márgenes quirúrgicos anteriormente descritos. La extracción tisular fue realizada en bloque respetando la integridad de la cápsula de la masa tumoral. El resultado de la evaluación histopatológica de la masa reveló la presencia de un fibrosarcoma cutáneo.

\section{Manejo anestésico}

Un catéter 20 G (Catéter IV, Nipro Medical Corporation, Osaka, Japón) fue colocado en la vena cefálica derecha para administrar $5 \mathrm{ml} / \mathrm{kg} / \mathrm{h}$ de ringer lactato (Solución lactato de ringer BP, Laboratorios ERMA S.A., Funza, Colombia). El paciente se premedicó con $0,01 \mathrm{mg} / \mathrm{kg}$ IV de acepromacina (Tranquilán ${ }^{\circledR}$, Laboratorios ZOO, Bogotá, 
Colombia), 0,3 mg/kg SC de meloxicam (Meloxic ${ }^{\circledR}$ 0,5\%, Provet, Medellín, Colombia) y $3 \mathrm{mg} / \mathrm{kg}$ IV de tramadol (Tramadol, Genfar ${ }^{\circledR}$, Cali, Colombia). Treinta minutos después, la anestesia se indujo con $5 \mathrm{mg} / \mathrm{kg}$ IV de propofol (Propofol 1\% MCT/LCT, Fresenius Kabi, Graz, Austria). Después de la intubación traqueal, la anestesia se mantuvo inicialmente con isoflurano (ISO) al 3\% (Isoflurano USP, Piramal Healthcare Ltd, Digwal, India) en oxígeno al $100 \%$ en un circuito anestésico circular con ventilación espontánea. El flujo inicial de $\mathrm{O} 2$ se administró a razón de $50 \mathrm{ml} / \mathrm{kg} /$ minuto durante 10 minutos; posteriormente, el flujo de gas fresco se disminuyó a 10 $\mathrm{ml} / \mathrm{kg} /$ minuto durante el procedimiento quirúrgico. La concentración alveolar mínima (CAM) del ISO sería ajustada en el vaporizador según el plano anestésico obtenido en el paciente. El monitoreo anestésico se realizó con un monitor multiparamétrico (Monitor iM12E, Shenzhen Biocare Electronics Co. Ltd., Shenzhen, China) en función de la frecuencia cardiaca $(\mathrm{FC})$, ritmo electrocardiográfico en derivación II, frecuencia respiratoria (FR), saturación de oxígeno de la hemoglobina ( $\mathrm{SpO} 2)$, tensión de dióxido de carbono espirado (EtCO2) y presión arterial media (PAM) de la arteria tibial craneal derecha por método oscilométrico.

\section{Bloqueo de los nervios intercostales}

Una vez en plano anestésico y preparada asépticamente la piel de ambos hemitórax, se posicionó la paciente en decúbito lateral derecho. Los espacios intercostales de T6-T11 fueron localizados por palpación digital de caudal a craneal. Una aguja espinal $22 \mathrm{G}$ de 31/2 pulgadas (Spinocan, B/Braun, Melsungen, Alemania) fue acoplada a una extensión anestésica y a una jeringa previamente cargada con $3 \mathrm{ml}$ de bupivacaína (Bupirov ${ }^{\circledR}$ 0,5\%, Lab. Ropsohn Therapeutics Ltda, Bogotá, Colombia) y $1 \mathrm{ml}$ de aire. La aguja se introdujo a través de la piel para luego ser dirigida hacia el aspecto caudal de la onceava costilla, posteriormente esta se redirigió hacia medial hasta percibir pérdida de la resistencia según la técnica descrita por Read \& Schroeder (2013). En este punto se realizó la técnica de succión para descartar una punción vascular no intencionada. Se evaluó la ausencia de resistencia a la inyección observando la compresión generada en la burbuja de aire contenida en la jeringa. En caso de percibir resistencia la aguja sería reposicionada y se repetiría el procedimiento. Finalmente se inyectaron lentamente 0,5 $\mathrm{ml}$ de bupivacaína por cada nervio. Al finalizar la inyección del anestésico local se retiró la aguja y se realizó el procedimiento para los nervios intercostales T10-T6, para luego continuar con el mismo procedimiento en el hemitórax izquierdo. Una vez realizado el último bloqueo, se esperó 20 minutos antes de iniciar el procedimiento quirúrgico.

\section{Monitoreo anestésico intraoperatorio}

Un incremento de la FC o la PAM por encima del $20 \%$ respecto al valor preincisional fueron considerados como indicativo de una cobertura analgésica insuficiente, en cuyo caso se administraría como analgesia de rescate $3 \mu \mathrm{g} / \mathrm{kg}$ IV de fentanilo. El tiempo total de la anestesia fue de 110 minutos durante los cuales las variables hemodinámicas se mantuvieron estables. Las variables cardiorrespiratorias y anestésicas evaluadas se muestran en la tabla 1. 
Tabla 1. Valores de intraoperatorios de frecuencia cardiaca, frecuencia respiratoria, presión arterial media, saturación de oxígeno de la hemoglobina, tensión de dióxido de carbono espirado y concentración alveolar mínima de isoflurano ajustada en el vaporizador, de un canino sometido a escisión quirúrgica de una masa tumoral ubicada en la pared abdominal cráneo-ventral.

\begin{tabular}{|c|c|c|c|c|c|c|}
\hline & $\begin{array}{c}\mathrm{FC} \\
(\mathrm{l} / \mathrm{m})\end{array}$ & $\begin{array}{c}\text { FR } \\
(\mathrm{v} / \mathrm{m})\end{array}$ & $\begin{array}{c}\text { PAM } \\
(\mathrm{mmHg})\end{array}$ & $\begin{array}{c}\text { SpO2 } \\
(\%)\end{array}$ & $\begin{array}{l}\text { EtCO2 } \\
(\mathrm{mmHg})\end{array}$ & $\begin{array}{c}\text { CAM ISO } \\
(\%)\end{array}$ \\
\hline $\begin{array}{c}\text { Valor } \\
\text { preincisional }\end{array}$ & 100 & 29 & 80 & 95 & 40 & 3,0 \\
\hline $\begin{array}{c}\text { Valor } \\
\text { promedio }\end{array}$ & 90 & 16 & 73 & 96 & 39 & 2 \\
\hline $\begin{array}{l}\text { Límites } \\
\text { mín-máx }\end{array}$ & $80-104$ & $14-20$ & $63-83$ & $95-99$ & $35-41$ & $1,3-3,0$ \\
\hline
\end{tabular}

\section{Evaluación del dolor postoperatorio}

La recuperación anestésica fue libre de complicaciones. Después de la extubación se evaluaron la FC, la FR, y la PAM de la arteria tibial craneal derecha por método oscilométrico, por medio de un monitor multiparamétrico (Monitor iM12E, Shenzhen Biocare Electronics Co. Ltd., Shenzhen, China). De igual manera, se evaluó la escala de medición compuesta del dolor de Glasgow (CMPS-SF) cada hora, durante seis horas por el mismo observador (Tabla 2). Valores superiores a 6 en esta escala serían considerados como indicativo de cobertura analgésica insuficiente en cuyo caso se administraría analgesia de rescate con $0,5 \mathrm{mg} / \mathrm{kg}$ IM de morfina. Una semana después se evidenció una adecuada cicatrización de la herida y de la capacidad ventilatoria de la paciente (Figura 2). 
Tabla 2. Valores postoperatorios de frecuencia cardiaca, frecuencia respiratoria, presión arterial media y de la escala de medición compuesta del dolor de Glasgow, de un canino sometido a escisión quirúrgica de una masa tumoral ubicada en la pared abdominal cráneo-ventral.

\begin{tabular}{ccccc}
\hline Hora & $\begin{array}{c}\text { FC } \\
(\mathrm{l} / \mathrm{m})\end{array}$ & $\begin{array}{c}\text { PAM } \\
(\mathrm{mmHg})\end{array}$ & $\begin{array}{c}\text { FR } \\
(\mathrm{V} / \mathrm{m})\end{array}$ & CMPS-SF \\
\hline 1 & 90 & 85 & 20 & 2 \\
\hline 2 & 92 & 80 & 22 & 1 \\
\hline 3 & 88 & 79 & 18 & 1 \\
\hline 4 & 86 & 80 & 18 & 1 \\
\hline 5 & 89 & 78 & 17 & 19 \\
\hline FC (Vm): frecuenca cardiaca (latidos/minuto), PAM (mmHg): presión arterial media (milimetros de mercurio): FR (v/m): \\
frecuencia res piratoria (ventilaciones/minuto), CMPS-SF: escala de medición compuesta del dolor de Glasgow.
\end{tabular}

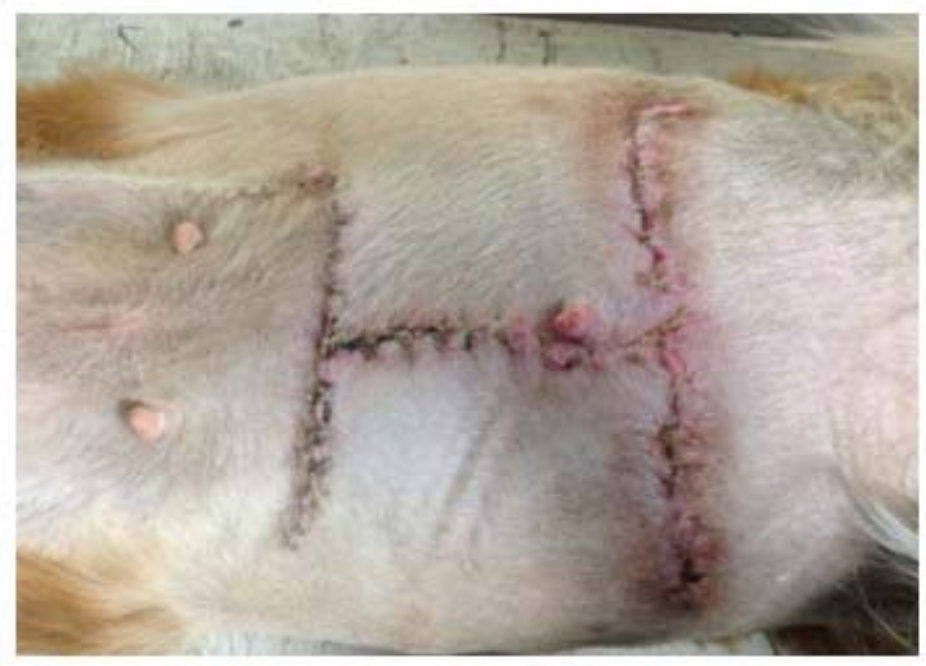

Figura 2. Representación del tamaño de las incisiones

quirúrgicas realizadas.

\section{Discusión}

El presente reporte de caso sugiere que el bloqueo anestésico de los nervios intercostales T6-T11 por medio de la técnica de marcas anatómicas de superficie como parte de un protocolo de anestesia multimodal proporcionó una adecuada antinocicepción intraoperatoria y una óptima analgesia postoperatoria para la escisión quirúrgica de una masa tumoral ubicada la pared abdominal cráneo-ventral en el 
paciente evaluado, lo cual se evidenció por medio de estabilidad en los parámetros cardiovasculares evaluados y el puntaje de la escala de medición del dolor de Glasgow.

La incapacidad de suministrar una analgesia segura y eficaz durante y después de la realización de la cirugía abdominal continúa siendo uno de los impedimentos para la introducción de técnicas de anestesia loco-regional en este tipo de intervenciones quirúrgicas (Finnerty et al., 2010). Una de las técnicas anestésicas más comúnmente empleadas en la realización de abordajes abdominales es el bloqueo epidural lumbosacro; no obstante, al realizar dicha técnica con un volumen convencional de 0,2 $\mathrm{ml} / \mathrm{kg}$ de bupivacaína al $0,25 \%$ se alcanza una cobertura analgésica únicamente en abordajes caudales al ombligo (Freire et al., 2010), lo cual limita su utilidad en procedimientos del abdomen craneal. Otero (2006) por su parte plantea que con un volumen de $1,5 \mathrm{ml}$ de bupivacaína al $0,25 \%$ por cada $10 \mathrm{~cm}$ de longitud de la médula espinal desde el hueso occipital hasta la primera vértebra coccígea se obtiene una cobertura analgésica hasta T5. Sin embargo, se debe considerar que para animales con un peso de hasta $30 \mathrm{~kg}$ el límite máximo de volumen a emplear para la epidural lumbosacra es de $6 \mathrm{ml}$ (Toske \& Dyson, 2000). En cuyo caso, para efectos del paciente evaluado el volumen final sería de $7,5 \mathrm{ml}$, contrariando el volumen final recomendado, razón por la cual no se empleó este bloqueo anestésico. También se debe considerar que dicha técnica está contraindicada en pacientes con coagulopatías, hipotensión, infecciones cutáneas en el sitio de punción, trauma de cadera con alteración anatómica de la misma (Aarnes, 2016); además, según la experiencia de los autores, la técnica se dificulta en pacientes con obesidad mórbida como el estudiado. Todo esto puede limitar en un momento dado la utilidad de esta técnica anestésica, en cuyo caso el bloqueo continuo con catéter epidural torácico podría ser más efectivo en procedimientos craneales al ombligo (Franci et al., 2012); sin embargo, dicho procedimiento es técnicamente más complejo; asimismo, este bloqueo está sujeto a complicaciones como el síndrome de Horner, depresión respiratoria, déficit propioceptivo, vomito, entre otras (Franci et al., 2012). Debido a estas limitaciones, el TAP block se consolida como una técnica alternativa de anestesia loco-regional que ha despertado gran interés en la anestesiología veterinaria al proveer analgesia local de la pared abdominal (Schroeder et al., 2011; Bruggink et al., 2012; Portela et al., 2014; Drozdzynska \& Viscallas, 2015). Este bloqueo es realizado inyectando un anestésico local en el plano facial existente entre el músculo oblicuo abdominal interno y el transverso abdominal, sitio por donde discurren los ramos ventrales de los nervios T11-L3, los cuales inervan la pared abdominal a manera de plexo (Schroeder et al., 2011). Diferentes estudios realizados en anestesia humana han demostrado que el TAP block disminuye el consumo de gases anestésicos volátiles, el gasto de analgesia de rescate con opioides, y mejora el confort de los pacientes después de una cirugía abdominal (López et al., 2013; Parikh et al., 2013).

Sin embargo, dos estudios anatómicos realizados en cadáveres de perros que evaluaron la distribución de una tinción sobre los nervios diana del TAP block, sugieren que dicho bloqueo no proporcionaría analgesia para el aspecto más craneal de la pared abdominal, toda vez que en estos estudios la tinción se distribuyó cranealmente hasta T11 tan solo en el 20\% de los casos (Schroeder et al., 2011). Para Hermason (2013) la inervación de la pared abdominal craneal está dada por los nervios intercostales T9-T13, los nervios lumbares iliohipogástricos e ilioinguinal y ocasionalmente por el T8. Lo cual indica que para realizar procedimientos quirúrgicos en la pared abdominal craneal se debe suplementar el bloqueo del TAP con otro tipo de bloqueo loco-regional (Portela et al., 
2014), o incluso reemplazarlo por otras técnicas como el BNI (Echeverry et al., 2015). Recientemente se ha descrito un estudio anatómico en el perro que evaluó la distribución de una tinción aportada por el TAP realizado desde un abordaje subcostal evidenciando una adecuada tinción de los ramos de T9-T13 (Drozdzynska \& Viscallas, 2015); sin embargo, se deben realizar estudios clínicos aleatorizados para validar su aplicabilidad.

Los ramos ventrales de los nervios intercostales T2-T12 proveen la inervación sensorial de la piel del aspecto latero-ventral del tórax, así como de la región cráneo-ventral abdominal (Read \& Schroeder, 2013). Al bloquear los ramos ventrales de los nervios intercostales T2-T11 se garantiza a su vez un bloqueo de los ramos cutáneo lateral, muscular distal y cutáneo ventral (T11 carece de su ramo cutáneo ventral lo cual se compensa con un alargamiento de su ramo cutáneo lateral que alcanza la línea media torácica y abdominal) (Evans \& de Lahunta, 2013). Esto resalta la importancia de dicho bloqueo loco-regional en los procedimientos quirúrgicos que comprenden la pared abdominal craneal al poner de manifiesto el componente sensitivo de dicha región anatómica.

Las escisiones de masas tumorales generan descargas nociceptivas considerables dependiendo de su grado de invasividad (Fox, 2014), lo cual puede aumentar la CAM de los gases anestésicos, el consumo de analgesia de rescate con opioides, y los valores de los parámetros hemodinámicos intraoperatorios. Por este motivo, la selección de los nervios T6-T11 se hizo teniendo en cuenta la superposición de los dermatomas asociados (Evans \& de Lahunta, 2013), bloqueando dos nervios craneales y dos nervios caudales a los dermatomas que inervaban el área donde estaba localizada la masa tumoral.

En la actualidad se ha planteado la actividad del tono parasimpático como método eficaz para detectar la sensibilidad intraoperatoria en los perros (Pedroza et al., 2015). Sin embargo, dicha tecnología presenta un elevado costo y en la mayoría de los casos tendría aplicabilidad únicamente en el área investigativa. Por lo tanto, el clínico debe basarse en métodos de monitoreo analgésico más prácticos y de fácil consecución como el monitoreo de la PAM por método oscilométrico y el comportamiento de la FC (Höglund et al., 2011; De Marzo et al., 2012; Voigt et al., 2013; Sarotti et al., 2015). Recientemente Mangas et al. (2016) demostraron que el registro de la actividad del tono parasimpático se correlaciona con el comportamiento de la PAM y la FC, lo cual sugiere que en los casos en que no se pueda disponer de dicha tecnología, monitorear la PAM y la FC resulta adecuado para detectar pobres coberturas analgésicas en perros.

Experimentalmente se ha demostrado que realizar procedimientos quirúrgicos que supongan una alta estimulación nociceptiva propicia el empleo de una alta CAM de ISO obteniendo una fracción espirada del mismo (EtISO) de hasta 2,04\% (Figueiró et al., 2016). Es de mencionar que el EtISO es el valor real de las concentraciones de ISO a nivel cerebral (Burns, 2015); en ese caso, cuando no se realiza un monitoreo de EtISO el consumo del gas anestésico es mandatorio por el juicio subjetivo del anestesista, lo cual abriría la posibilidad de ajustar la CAM de ISO en el vaporizador a valores posiblemente superiores a $3 \%$ en procedimientos dolorosos, según la experiencia de los autores. De igual manera, se debe aclarar que cuando se emplean sistemas anestésicos cerrados, con protocolos de bajos flujos de gas fresco (O2 $10 \mathrm{ml} / \mathrm{kg} / \mathrm{minuto}$ ) como en el presente caso, para obtener un EtISO de 1,3\% en el perro, se debe ajustar el vaporizador 
con valores que podrían llegar a ser incluso superiores a 2\% (Lachin \& Bufalari, 2012; Burns, 2015), lo cual está sujeto a la alta probabilidad de reinhalación del gas anestésico previamente espirado (Steffey et al., 2015). De esta forma, el realizar un amplio BNI permitió obtener una adecuada cobertura analgésica reflejada en una CAM de ISO promedio ajustada en el vaporizador de $2 \%$, unas constantes fisiológicas estables y el no requerimiento de rescate analgésico intraoperatorio. Esto sugiere que sin la aplicación de la técnica loco-regional empleada en este paciente, posiblemente los parámetros hemodinámicos evaluados hubieran sido superiores a los obtenidos, y por esta razón necesitando de valores más altos la CAM del ISO y de analgésicos de rescate intraoperatorios a fin de obtener una adecuada cobertura analgésica.

Por otro lado, la técnica loco-regional empleada permitió minimizar las manifestaciones comportamentales álgicas evaluadas con la escala CMPS-SF, prescindiendo del consumo de morfina postoperatoria, similar a lo reportado por Portela et al. (2014) en su ensayo clínico, y por Trębaczet al. (2011), quienes demostraron experimentalmente por medio de la tinción de los nervios diana, la cobertura que se puede alcanzar al realizar este bloqueo por medio de la técnica de marcas anatómicas de superficie.

La realización del BNI ha sido descrita empleando bupivacaína al 0,25\% (Portela et al., 2014); no obstante, ha sido demostrado que utilizar bupivacaína a concentraciones más altas como la empleada en este reporte $(0,5 \%)$, permite obtener bloqueos con una latencia más prolongada, además de minimizar la dosis total del anestésico local (Takeda et al., 2015). De esta forma, el volumen del anestésico local empleado en este paciente fue a razón de $0,015 \mathrm{ml} / \mathrm{kg}$ para cada nervio intercostal, contrario al empleado por Portela et al. (2014) $(0,03-0,04 \mathrm{ml} / \mathrm{kg})$, donde se empleó bupivacaína a una concentración menor. La dosis total que fue empleada para este paciente fue de 0,9 $\mathrm{mg} / \mathrm{kg}$, respetando los márgenes terapéuticos para la especie $(2 \mathrm{mg} / \mathrm{kg}$ ) (Pawson \& Forsyth, 2008).

Dentro de las complicaciones inherentes a la realización del bloqueo intercostal se encuentra la toxicidad por el anestésico local, punción vascular, neumotórax y anestesia espinal (Read \& Schroeder, 2013); sin embargo, ninguna de estas complicaciones se presentó en el paciente evaluado.

Se sugiere que el uso de anestesia loco-regional en cirugía oncológica permitiría reducir el estrés quirúrgico, el consumo de anestésicos volátiles y opioides y consecuentemente disminuir la inmunosupresión perioperativa, la angiogénesis, y en última estancia la recurrencia del cáncer. Sin embargo, no hay suficiente evidencia que permita confirmar esta hipótesis (Cata et al., 2014).

\section{Conclusión}

Para efectos de este caso clínico, la realización de bloqueo bilateral de nervios intercostales T6-T11 fue efectiva para proveer una adecuada analgesia perioperatoria, manteniendo los parámetros cardiovasculares estables y CAM de ISO baja durante el periodo operatorio, además de una puntuación mínima en la escala de Glasgow durante 
el periodo postoperatorio. La técnica de anestesia loco-regional utilizada en este paciente, pudiera ser empleada de manera segura y eficaz para proveer anestesia y analgesia durante la realización de procedimientos quirúrgicos realizados en la pared abdominal cráneo-ventral del perro. Esta técnica podría ser considerada como una alternativa frente al bloqueo epidural tradicionalmente realizado en procedimientos quirúrgicos que involucran la pared abdominal.

\section{Referencias bibliográficas}

- Aarnes, T.K. Epidurals and spinals. In: Lerche, P.; Aarnes, T.K.; Covey-Crump, G.et al. (Ed). Handbook of small animal regional anesthesia and analgesia techniques. New Jersey: Wiley-Blackwell, 2016. p. 75-82.

- Bruggink, S.M.; Schroeder, K.M.; Baker-Herman, T.L. et al. Weight-based volume of injection influences cranial to caudal spread of local anesthetic solution in ultrasoundguided transversus abdominis plane blocks in canine cadavers. Veterinary Surgery, v.41, n.4, p.455-457, 2012.

- Burns, P. Isoflurane and sevoflurane: application and protocols. Clinician's Brief, p.83$87,2015$.

- Cata, J.P.; Hernandez, M.; Lewis, V.O. et al. Can regional anesthesia and analgesia prolong cancer survival after orthopaedic oncologic surgery? Clinical Orthopaedics and Related Research, v.72, n.5, p.1434-1441, 2014.

- De Marzo, C.; Crovace, A.; De Monte, V. et al. Comparison of intra-operative analgesia provided by intravenous regional anesthesia or brachial plexus block for pancarpalarthrodesis in dogs. Research in Veterinary Science, v.93, n.3, p.1493-1497, 2012.

- Drozdzynska, M.; Viscallas, J. Descripción del bloqueo ecoguiado del plano transverso del abdomen con abordaje subcostal en el perro: estudio en cadáveres. In: XI Congreso Nacional de la Sociedad Española de Anestesia y Analgesia Veterinaria, 2015, Madrid, España. Memorias XI Congreso Nacional de la Sociedad Española de Anestesia y Analgesia Veterinaria; 2015, p.82.

- Echeverry, D.F.; Peláez, J.T.; Ospina, D.A. et al. Analgesic effect of a bilateral intercostal nerve block in a dog undergoing surgical excision of an abdominal Wall tumor. In: 12th World Congress of a Veterinary Anaesthesiology, 2015, Kyoto, Japan. Memorias 12th World Congress of a Veterinary Anaesthesiology; 2015, p.181. 
- Evans, H.E.; de Lahunta, A.; editores. Miller's Anatomy of the Dog. $4^{\mathrm{a}}$ ed. Filadelfia, Estados Unidos: Saunders, Elsevier; 2013. 850p.

- Figueiró, M.R.; Soares, J.H.; Ascoli, F.O. et al. Isoflurane MAC determination in dogs using three intensities of constant-current electrical stimulation. Veterinary Anaesthesia and Analgesia, v.43, n.5, p.464-471, 2016.

- Finnerty, O.; Carney, J., McDonell, J.G. Trunk blocks for abdominal surgery.Anaesthesia, v.65, p.76-83, 2010 (suppl.1).

- Fox, S.M.; editor. Pain management in small animal medicine. 1a ed. Florida, Estados Unidos: CRC Press Taylor \& Francis Group, 2014. 416p.

- Franci, P.; Leece, E.A.; Corletto, F. Thoracic epidural catheter placement using a paramedian approach with cephalad angulation in three dogs. Veterinary Surgery, v.41, n.7. p.884-889, 2012.

- Freire, C.D.; Torres, M.L.; Fantoni, D.T.; Calvancanti, R.L.; Noel-Morgan, J. Bupivacaine $0.25 \%$ and methylene blue spread with epidural anesthesia in dog.Veterinary Anaesthesia and Analgesia, v.37, n.1, p.63-69, 2010.

- Hellyer, P.; Rodan, I.; Brunt, J. et al. AAHA/AAFP pain management guidelines for dogs \& cats. Journal of American Animal Hospital Association, v.43, n.5, p.235-248, 2007.

- Hermanson, J.W. The muscular system. In: Evans, H.E.; de Lahunta, A. (Ed) Miller's Anatomy of the Dog. Philadelphia: Saunders, Elsevier, 2013. p. 185-276.

- Höglund, O.V.; Olsson, K.; Hagman, R. et al. Comparison of haemodynamic changes during two surgical methods for neutering female dogs. Research in Veterinary Science, v.91, n.1, p.159-163, 2011.

- Lachin, A.; Bufalari, A. Monitoraggio dei gas respirati. In: Bufalari, A.; Lachin, A. (Ed) Anestesia cane, gatto e animalinon convenzionali. Milano: Elsevier, 2012. p. 772789.

- López, J.M.; Jiménez, B.M.; Areán, I.et al. Bloqueo transverso abdominal ecoguiado vs. infiltración de herida quirúrgica en cirugía ambulatoria de hernia inguinal. Cirugía Mayor Ambulatoria, v.18, n.1, p.7-11, 2013.

- Mangas, M.T.; Lima, J.F.; Abellán, E. et al. Estudio prospectivo sobre el valor predictivo de la monitorización nociceptiva intraoperatoria de los cambios hemodinámicos en perros anestesiados: estudio piloto. In: X Southern European Veterinary Conference, 51 Congreso Nacional AVEPA, 2016, Granada, 
España. Memorias X Southern European Veterinary Conference, 51 Congreso Nacional AVEPA, 2016.

- Melzack, R.; Wall, P.D.; editores. Textbook of pain. $4^{\mathrm{a}}$ ed. London, England: Churchill Livingstone, 1999. 1588p.

- Otero, P. Epidural anesthesia and analgesia. In: 31st World Small Animal Veterinary Congress, 12th European Congress FECAVA, National Congress CSAVA, 2006, Prague, Czech Republic. Memorias 31st World Small Animal Veterinary Congress, 12th European Congress FECAVA, National Congress CSAVA; 2006, p.199-201.

- Parikh, B.K.; Waghmare, V.T.; Shah, V.R. et al. The analgesic efficacy of ultrasoundguided transversus abdominis plane block for retroperitoneoscopic donor nephrectomy: A randomized controlled study. Saudi Journal of Anaesthesia, v.7, n.1, p.43-47, 2013.

- Pawson, P.; Forsyth, S. Anesthetic agents. In: Maddison, J.E.; Page, S.; Church, D. (Ed). Small Animal Clinical Pharmacology. Filadelfia: Saunders, Elsevier, 2008. p. 83112.

- Pedroza, S.; Expósito, A.; Aguado, D. et al. Monitorización de la nocicepción intraoperatoria en perros mediante un monitor de tono parasimpático: estudio piloto. In: XI Congreso Nacional Sociedad Española de Anestesia y Analgesia Veterinaria, 2015, Madrid, España. Memorias XI Congreso Nacional Sociedad Española de Anestesia y Analgesia Veterinaria; 2015, p.88.

- Portela, D.A.; Romano, M.; Brignati, A. Retrospective clinical evaluation of ultrasound guided transverse abdominis plane block in dogs undergoing mastectomy. Veterinary Anaesthesia and Analgesia, v.41, n.3, p.319-324, 2014.

- Read, M.R.; Schroeder, C.A. The Trunk. In: Campoy, L.; Read, M.R. (Ed). Small animal regional anesthesia and analgesia. New Jersey: Wiley-Blackwell, 2013. p. 167198.

- Sarotti D, Rabozzi R, Franci P. Comparison of epidural versus intrathecal anaesthesia in dogs undergoing pelvic limb orthopaedic surgery. Veterinary Anaesthesia and Analgesia, v.42, n.4, p.405-413, 2015.

- Schroeder, C.A.; Snyder, L.B.; Tearney, C.C.et al. Ultrasound-guided transversus abdominis plane block in the dog: an anatomical evaluation. Veterinary Anaesthesia and Analgesia; v.38, n.3, p.267-271, 2011. 
- Steffey, E.P.; Mama, K.R.; Brosnan, R.J. Inhalation Anesthetics. In: Grimm, K.A.; Lamont, L.A.; Tranquilli, W.J. et al (Ed). Veterinary Anesthesia and Analgesia The Fifth Edition of Lumb and Jones. Iowa: Wiley-Blackwell, 2015. p. 297-331.

- Takeda, A.; Cunha, L.H.; Hosoi, A. et al. Concentración mínima efectiva de bupivacaína para el bloqueo del plexo braquial vía axilar guiado por ecografía. Revista Brasileira de Anestesiologia, v.65, n.3, p.163-169, 2015.

- Torske, K.E.; Dyson, D.H. Epidural analgesia and anesthesia. Veterinary Clinics of North America Small Animal Practice, v.30, n.4. p. 859-874, 2000.

- Trębacz, P.; Trębacz, E.; Jurka, P. et al. Comparison of the efficiency of anatomical and ultrasound-guided method for the Th4-Th7 intercostal nerve block in dogs. Bulletin of the Veterinary Institute in Pulawy, v.55, n.3, p.507-511, 2011.

- Voigt, A.M.; Bergfeld, C.; Beyerbach, M. et al. Effects of isoflurane with and without dexmedetomidine or remifentanil on heart rate variability before and after nociceptive stimulation at different multiples of minimum alveolar concentration in dogs. American Journal of Veterinary Research, v.74, n.5, p.665-671, 2013.

Cómo citar: Ospina-Argüelles, D.E.; Buriticá-Gaviria, E.F.; Echeverry-Bonilla, D.F. Bloqueo anestésico de los nervios intercostales T6-T11 en un canino sometido a escisión quirúrgica de una masa tumoral ubicada en la pared abdominal cráneo-ventral. Reporte de caso. Revista Veterinaria y Zootecnia, v. 11, n. 1, p. 83-95. DOI: 10.17151/vetzo.2017.11.1.7 